\title{
miR-100 inhibits the migration and invasion of nasopharyngeal carcinoma by targeting IGF1R
}

\author{
XIAOYAN SUN, XIAOYING LIU, YANMEI WANG, SHUQIN YANG, YAO CHEN and TIEJUN YUAN \\ Department of Otorhinolaryngology, Weifang People's Hospital, Weifang, Shandong 261041, P.R. China
}

Received November 29, 2017; Accepted March 12, 2018

DOI: $10.3892 / 01.2018 .8420$

\begin{abstract}
Nasopharyngeal carcinoma (NPC) is a cancer pattern that often develops in the epithelial cells of the nasopharynx. miR-100 is a miRNA that has been identified in a number of cancers. The aim of the present study was to investigate whether miR-100 can affect cell migration and proliferation of NPC by targeting insulin-like growth factor 1 receptor (IGF1R). Western blot analysis was used to determine the protein levels of genes. The reverse transcription-quantitative PCR (RT-qPCR) was used to detect the expression level of miR-100 and IGF1R. Transwell assay was used to detect the migration and invasion of cell lines. The luciferase reporter assay was employed to confirm the target gene of miR-100. miR-100 expression was highly reduced in NPC tissues compared with non-cancerous tissues. Overexpression of miR-100 significantly inhibited the migration and invasion of NPC cell lines C666-1 and SUNE1. IGF1R was a downstream target of miR-100 and was downregulated by miR-100. Knockdown of IGF1R by siRNA suppressed cell proliferation of the C666-1 cell line. The newly identified miR-100/IGF1R axis offers novel biomarkers for the therapeutic intervention of NPC treatment. As a result, our findings suggest that miR-100 plays an important role in suppressing migration and invasion of NPC cells and suppresses IGF1R expression by directly targeting its 3'-UTR. It is suggested that miR-100 may be a novel therapeutic target of microRNA-mediated suppression of cell migration and invasion in NPC. However, the role of
\end{abstract}

Correspondence to: Dr Yao Chen, Department of Otorhinola ryngology, Weifang People's Hospital, 151 Guangwen Street, Kuiwen, Weifang, Shandong 261041, P.R. China

E-mail: sdct921462@126.com

Abbreviations: miRNAs, microRNAs; NPC, nasopharyngeal carcinoma; EBV, Epstein-Barr virus; OSCC, oral squamous cell carcinoma; MTT, 3-[4, 5-di-methylthi-azol-2-yl]-2,5-diphenyltetrazolium bromide; IGF1R, insulin-like growth factor 1 receptor; CCRCC, clear cell renal cell cancer; VHL, von Hippel-Lindau; RT-qPCR, reverse transcription-quantitative PCR

Key words: IGF1R, invasion, migration, miR-100 the miR-100/IGF1R axis in NPC progression needs further investigation.

\section{Introduction}

Nasopharyngeal carcinoma (NPC) is a cancer pattern that often develops in epithelial cells of nasopharynx (1). The tumorigenesis of NPC has a number of causes, including genetic alternation, environmental factors and virus infection (2). Most NPCs are associated with Epstein-Barr virus (EBV) infection, and EBV genome can be detected in approximately $90 \%$ of NPC tissues (3). NPC is the 24 th most common cancer type worldwide. It is estimated that there were approximately 84,400 newly diagnosed cases and 51,600 deaths during 2008 (4). NPCs are categorized into three main types according to the differentiation degree based on the World Health Organization classification. The three main types are keratinizing, non-keratinizing and Basaloid carcinoma (5). Therefore, the development of new therapeutic interventions for NPCs is imperative.

MicroRNAs (miRNAs) are small non-coding RNAs, which are 20-22 nucleotides long (6). miRNAs regulate the expression of target genes by binding to 3'-UTR at the post-transcriptional level $(7,8)$. At present, a number of miRNAs have been identified with a dysfunctional role in various types of cancer. Such miRNAs function as a key inducer in tumor initiation and development (9). miR-100 is a miRNA that has been identified in a number of cancers. Henson et al (10) reported that miR-100 markedly inhibited the cell migration and invasion of oral squamous cell carcinoma (OSCC). miR-100 also regulates gene expression, including genes involved in radioresistant OSCC (10). miR-100 is involved in the modulation of G1/S transition and inhibits the terminal differentiation of acute myeloid leukemia (11). RBSP3 often functions as a tumor suppressor (12). miR-100 promotes the cell proliferation of acute myeloid leukemia by targeting RBSP3 (11). However, the potential mechanism of miR-100 in NPC is unclear.

The insulin-like growth factor pathway plays an important role in cell differentiation and apoptosis (13). Insulin-like growth factor 1 receptor (IGF1R) is one component of this signaling pathway (14). IGF1R is a transmembrane receptor, which is a tyrosine kinase. It is comprised of two $\beta$ subunits and two $\alpha$ subunits (15). IGF1R is involved in a number of diseases. IGF1R expression is highly increased in non-small 
cell lung cancer cells compared with corresponding normal cells. In addition, IGF1R expression is associated with EGFR expression (16). Yuen et al reported that IGF1R expression is significantly higher in clear cell renal cell cancer (CCRCC) than normal kidney cells and is negatively correlated with von Hippel-Lindau (VHL) expression (17). Nevertheless, the function of IGF1R in NPC remains unclear.

In the present study, we investigated the expression of miR-100 and IGF1R and their function in NPC. We identified that miR-100 expression was significantly reduced in NPC cells compared with corresponding non-cancerous cells, detected by reverse transcription-quantitative PCR (RT-qPCR). Overexpression of miR-100 significantly suppressed the migration and invasion of NPC cells. IGF1R was a downstream target of miR-100, as confirmed by luciferase reporter assay. IGF1R expression was highly increased in NPC cells compared with non-tumorous cells. Knockdown of IGF1R by siRNA significantly inhibited NPC cell proliferation, which was confirmed by western blot and MTT assays.

\section{Materials and methods}

Cell lines and patient samples. A total of 115 pairs of NPC tissues and their corresponding non-cancerous tissues were collected from patients who underwent NPC surgery between January 2015 and March 2017 in the Weifang People's Hospital (Weifang, China). The collected tissues were preserved in liquid nitrogen. None of the patients had received radiotherapeutic treatment before tissues were collected. Patients provided informed consent for the use of tissues in this study. The study was approved by the Ethics Committee of Weifang People's Hospital.

NPC cell lines C666-1 and SUNE1 were purchased from the American Type Culture Collection (Manassas, VA, USA). An NP460 normal nasopharyngeal epithelium cell line was used as the control. Cell lines were cultured in RPMI-1640 medium, which contained fetal bovine serum.

Plasmid construction and cell transfection. miR-100 mimic was synthesized and purchased from Thermo Fisher Scientific, Inc. (Waltham, MA, USA). miR-100 mimic was used to overexpress miR-100. siRNA for IGF1R was synthesized to knock down IGF1R gene.

The vectors (including miR-100 mimic and siRNA-IGF1R associated, which served as the negative control) were transfected into the cell lines using Lipofectamine 2000 Reagent (Tiangen Biotech Co., Ltd., Beijing, China) following the manufacturer's protocol.

Western blot assay. Tissue proteins were extracted using ProteinExt Mammalian Total Protein Extraction kit(TransGen Biotech Co., Ltd., Beijing, China). Protein concentration was detected using the Bradford assay. Proteins were separated on 12\% SDS-PAGE gel using Bio-Rad Mini-PROTEAN Tetra instrument (Bio-Rad Laboratories, Inc., Hercules, CA, USA). Then, proteins were electrotransferred onto polyvinylidene difluoride membranes. The membranes were incubated with primary antibodies against IGF1R and GAPDH (Sigma-Aldrich, St. Louis, MO, USA). Signals were visualized on Bio-Rad Gel Doc XR instrument (Bio-Rad Laboratories, Inc.).

$R T-q P C R$. Total RNAs of tissues were extracted using the EasyPure RNA kit (TransGen Biotech Co., Ltd.). RNAs were confirmed to be eligible using agarose gel electrophoresis. Then RNAs were inverse transcribed to single-strand cDNA using EasyScript First-Strand cDNA Synthesis SuperMix (TransGen Biotech Co., Ltd.). miR-100 expression was normalized by the U6 expression level and GAPDH expression was used as an internal control for IGF1R. Experiments were performed three times.

Luciferase reporter assay. Target Scan online tool (http://www. targetscan.org/vert_71/) was used to search the potential target of miR-100 in human. IGF1R was demonstrated as a downstream target of miR-100 and binds to 5602-5609 bp at 3'-UTR of IGF1R. In order to confirm this prediction, Psicheck ${ }^{\mathrm{TM}}-2$ vector was used in this study. The 3'-UTR of IGF1R was cloned and inserted into Psicheck ${ }^{\mathrm{TM}}-2$ plasmid (Psicheck $\left.{ }^{\mathrm{TM}}-2-\mathrm{WT}\right)$. The binding site was also mutated and inserted into Psicheck ${ }^{\mathrm{TM}}-2$ plasmid (Psicheck ${ }^{\mathrm{TM}}-2-\mathrm{MT}$ ). The Dual-Glo Luciferase Assay System (Promega Corporation, Madison, WI, USA) was used to measure luciferase activity.

Migration and invasion assay. Transwell assay was used to measure cell migration and invasion. Cells $\left(3 \times 10^{4}\right)$ were placed onto the top component of one chamber (Corning Life Sciences, Manassas, VA, USA) (pore size, $8 \mu \mathrm{m}$ ). The lower chamber was filled with serum-free medium. The chamber was cultured for $48 \mathrm{~h}$ using migration assay. Extracellular matrix gel was used for the cell invasion assay. Finally, migration and invasion cells were stained with crystal violet. Results were visualized using a light microscope (Olympus Corporation, Tokyo, Japan).

Proliferation assay. The 3-[4, 5-di-methylthi-azol-2-yl]-2,5-diphenyl-tetrazolium bromide (MTT) assay was used to detect cell proliferation. Cell suspensions of $100 \mu \mathrm{l}$ were added to each well. The 96-well plate was incubated at $5 \% \mathrm{CO}_{2}$ and $37^{\circ} \mathrm{C}$ for $48 \mathrm{~h}$. Then, $10 \mu \mathrm{l} \mathrm{MTT}$ solution $(5 \mathrm{mg} / \mathrm{ml})$ was added to each well and cultured for $4 \mathrm{~h}$. The crystals were dissolved in $150 \mu \mathrm{l}$ DMSO and absorbance at $490 \mathrm{~nm}$ was measured using an Eppendorf BioSpectrometer ${ }^{\circledR}$ kinetic instrument (Eppendorf, Hamburg, Germany).

Statistical analysis. Experiments in the present study were performed at least three times. Experimental results were presented as mean \pm SD. The SPSS 16.0 (SPSS, Inc, Chicago, IL, USA) software was used for data analysis comparisons were made using Student's t-test and one-way ANOVA post hoc test. A $\mathrm{P}<0.05$ was considered to indicate a statistically significant difference.

\section{Results}

miR-100 expression is significantly decreased in NPC tissues. In the present study, we investigated the miR-100 expression level in NPC and normal cells. miR-100 expression level was significantly decreased in 115 pairs of NPC tissues compared 
A

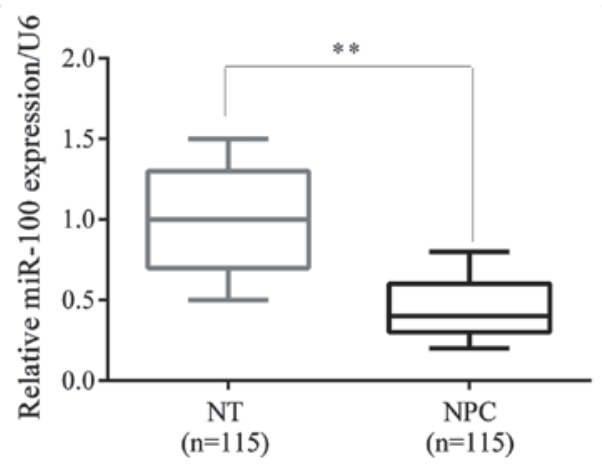

B

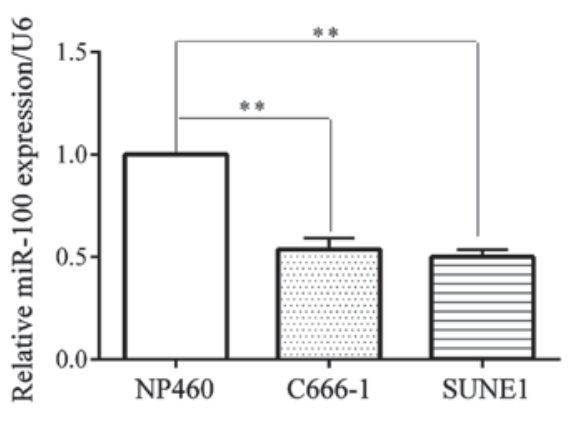

Figure 1. miR-100 is downregulated in NPC cell lines and tissues. (A) miR-100 expression was significantly decreased in NPC tissues compared with non-tumorous tissues. RT-qPCR was carried out in 115 pairs of NPC tissue samples. (B) miR-100 expression was significanlty decreased in NPC C666-1 and SUNE1 cell lines compared with normal nasopharyngeal cell line NP460 by RT-qPCR. NPC, nasopharyngeal carcinoma; RT-qPCR, reverse transcription-quantitative PCR. ${ }^{* *} \mathrm{P}<0.01$.

A

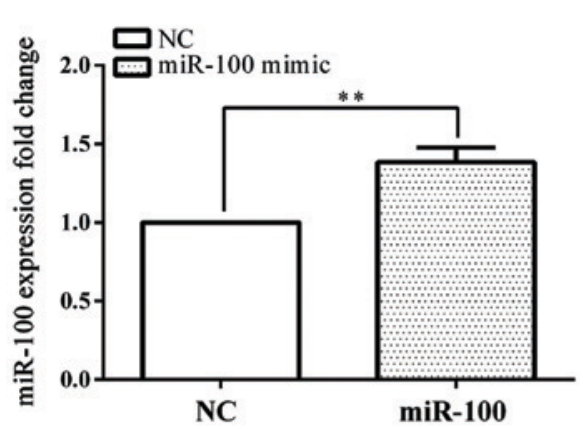

B

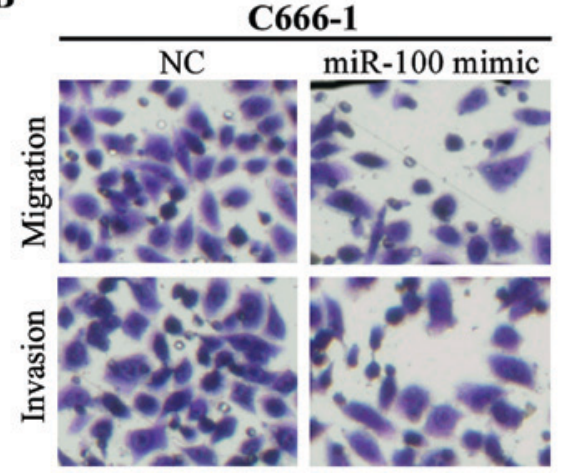

SUNE1

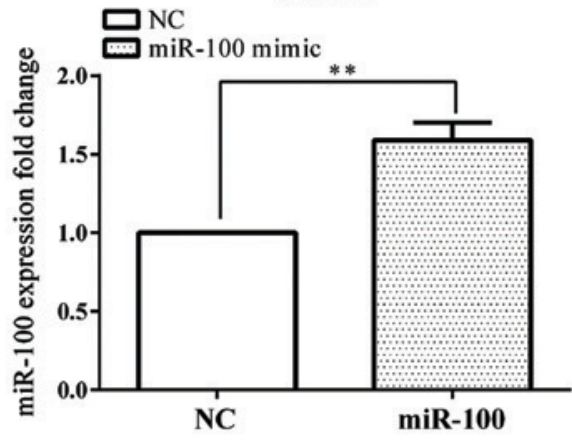

SUNE1

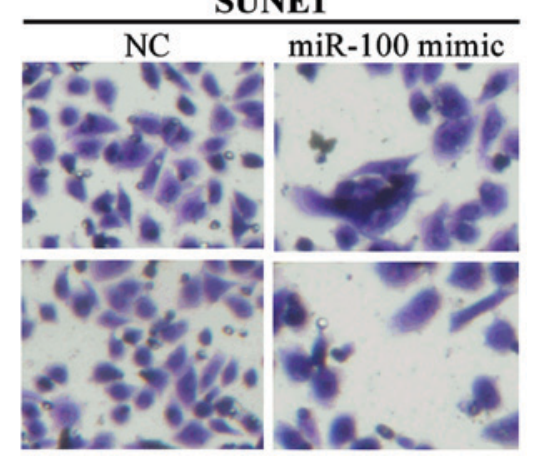

Figure 2. Overexpression of miR-100 significantly inhibits cell migration and invasion of NPC cell lines. (A) Overexpression of miR-100 in NPC C666-1 and SUNE1 cell lines was verified by RT-qPCR. (B) The inhibitory effects of the overexpression of miR-100 on cell migration and invasion were confirmed by Transwell assay. NPC, nasopharyngeal carcinoma; RT-qPCR, reverse transcription-quantitative PCR. ${ }^{* *} \mathrm{P}<0.01$.

with non-cancerous tissues by RT-qPCR (Fig. 1A). We also investigated the miR-100 expression levels of NPC C666-1 and SUNE1 cell lines and the normal NP460 nasopharyngeal epithelium cell line. The data showed that the miR-100 expression level was significantly suppressed in NPC cell lines compared with, the NP460 normal nasopharyngeal cell line (Fig. 1B).

Overexpression of miR-100 significantly inhibits cell migration and invasion in vitro. Transwell assay was used to investigate the effect of miR-100 on cell migration and invasion. miR-100 mimic was used to overexpress miR-100 in
C666-1 and SUNE1 cell lines. The overexpression of miR-100 was confirmed by RT-qPCR (Fig. 2A).

Overexpression of miR-100 stably suppressed C666-1 and SUNE1 cell migration and invasion by Transwell assay (Fig. 2B). These results showed that the overexpression of miR-100 inhibited NPC cell invasion and migration.

IGF1R is a downstream target of miR-100 and is downregulated by $m i R-100$. IGF1R was predicted as a potential target of miR-100 by the online Target Scan tool (http://www.targetscan. org/vert_71/) (Fig. 3A). We constructed Psicheck ${ }^{\mathrm{TM}}-2-\mathrm{WT}$ and Psicheck ${ }^{\mathrm{TM}}-2-\mathrm{MT}$ plasmid. NPC C666-1 and SUNE1 
$\mathbf{A}$

\begin{tabular}{|c|c|c|}
\hline \multicolumn{3}{|c|}{ Site: 5602-5609 of IGF1R } \\
\hline & 5' ...UUAAGUCCAGUAGAUUACGGGUA... & $3^{\prime}$ \\
\hline miR-100 & 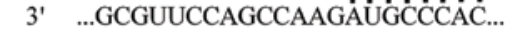 & $5^{\prime}$ \\
\hline MT UTR & $5^{\prime} \quad \ldots$..UAAGUCCAGUAGAUAUGCCCAC... & $3^{\prime}$ \\
\hline
\end{tabular}

B

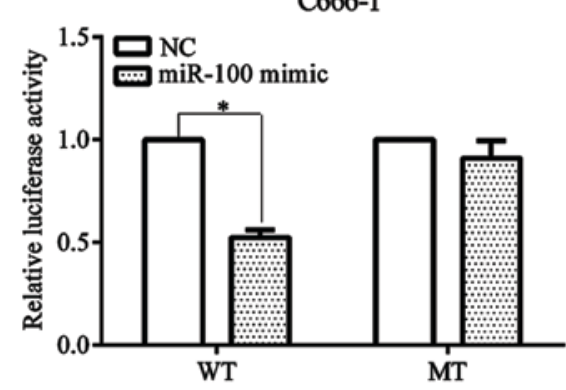

$\mathrm{C}$

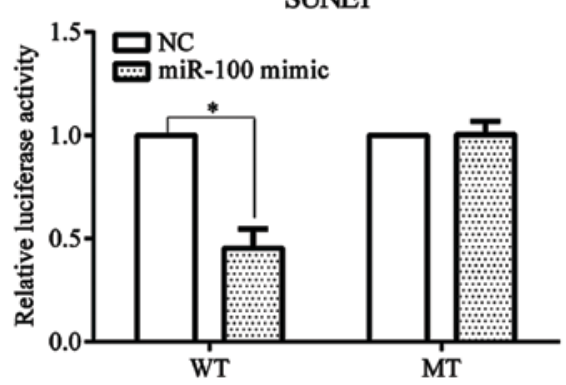

Figure 3. IGF1R is a downstream target of miR-100 and is downregulated by miR-100. (A) The binding sites of IGF1R for miR-100 was at 5602-5609 bp in 3'-UTR of IGF1R, which was predicted by TargetScan. Mutated nucleotides are denoted in red. (B) Luciferase activity of the C666-1 cell line was detected. (C) IGF1R protein level of SUNE1 cell line was detected by western blot assay. IGF1R, insulin-like growth factor 1 receptor. "P<0.05.

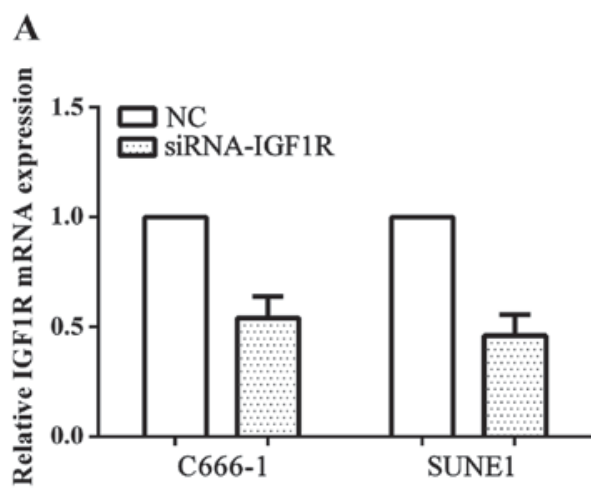

B

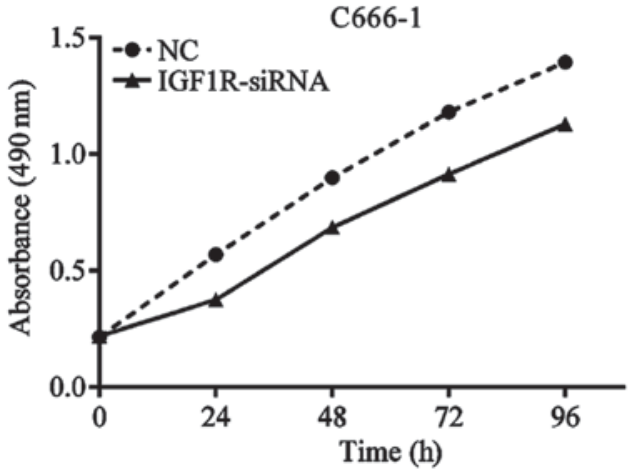

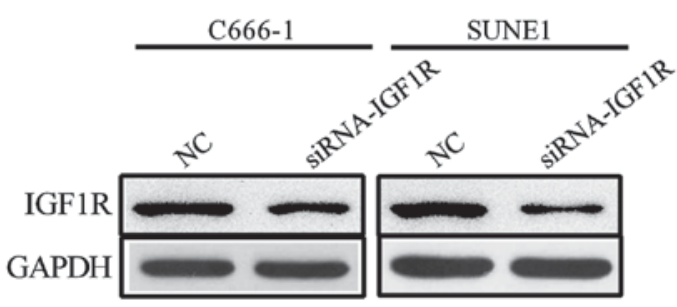

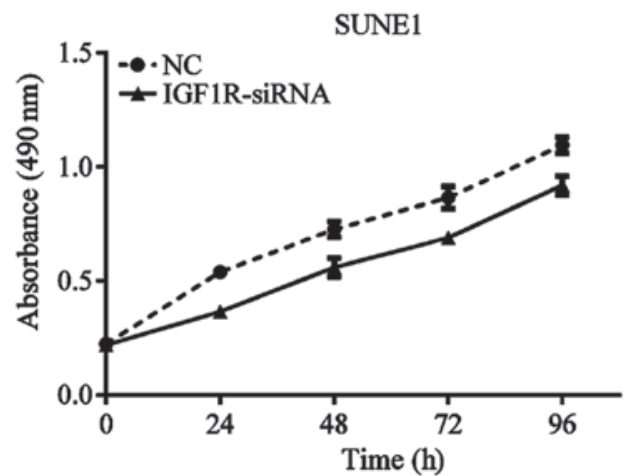

Figure 4. Knockdown of IGF1R significantly inhibits cell proliferation in vitro. (A) Cell proliferation of C666-1 and SUNE1 was detected by MTT assay. (B) IGF1R protein levels in C666-1 and SUNE1 cell lines were determined. IGF1R, insulin-like growth factor 1 receptor; MTT, 3-[4, 5-di-methylthi-azol2-yl]-2,5-diphenyl-tetrazolium bromide.

cells were co-transfected with miR-100 mimic or the negative control and Psicheck ${ }^{\mathrm{TM}}-2-\mathrm{WT}$ or Psicheck ${ }^{\mathrm{TM}}-2-\mathrm{MT}$ plasmid. Luciferase activity was significantly decreased when the cell line was co-transfected with miR-100 mimic and Psicheck ${ }^{\mathrm{TM}}-2-\mathrm{WT}$ plasmid compared with the negative control. The reduced effect disappeared when the cell line was co-transfected with miR-100 mimic and Psicheck ${ }^{\mathrm{TM}}-2-\mathrm{MT}$ plasmid (Fig. 3B).
Knockdown of IGF1R inhibits cell proliferation of NPC cell lines. In order to better understand the mechanism of IGF1R on NPC development, siRNA for IGF1R was used to knockdown the expression of IGF1R. The NPC C666-1 and SUNE1 cell lines were transfected with siRNA-IGF1R or negative control. The IGF1R mRNA and protein level was significantly decreased in cell lines transfected with siRNA compared with the negative control (Fig. 4A). In addition, the proliferation of cell lines 
transfected with siRNA were significantly reduced compared with the cell lines transfected with the negative control (Fig. 4B).

\section{Discussion}

NPC is a rare cancer type compared with other high incidence cancers. However, in specific areas, including southeastern Asia, Singapore, Vietnam, and Philippines, NPC has a markedly high incidence $(18,19)$. NPC tumorigenesis is complicated and remains unclear.

In the present study, we investigated the mechanism of miR-100 and the effect of IGF1R on NPC. We identified that miR-100 was highly downregulated in NPC cell lines compared with the normal cell lines and NPC tissues compared with non-tumorous tissues. In addition, the overexpression of miR-100 following transfection of the mimic into the two cell lines significantly inhibited migration and invasion. Many miRNAs have been reported to play an important role in the development of NPC. miR-BART22 is a newly identified EBV miRNA and can be detected in NPC. Overexpression of miR-BART22 may promote tumor cell invasion and proliferation. MAP3K5 is a target of miR-BART22 and is downregulated by miR-BART22 (20). In cisplatin-resistant NPC cells, miR-10b expression is increased in HNE1 cell lines. Overexpression of miR-10b promotes epithelial-mesenchymal transition (21). Cheung et al reported that miR-183 and miR-86 expression were decreased in NPC spheroids (22). In addition, transfection of miR-183 into cell lines suppressed tumor growth $(21,22)$. miRNAs have diverse effects on NPC development. Some miRNAs inhibit NPC cell growth; on the other hand, some miRNAs promote NPC cell growth. miR-15a demonstrates the suppressive effect on NPC cells. Overexpression of miR-15a significantly inhibits cell growth and triggers cell apoptosis (23). Few reports are available on miR-100. miR-100 has been investigated in oral cancer (10), acute myeloid leukemia (11) and endometrioid endometrial carcinoma (24).

IGF1R is important in the functional transformation of many oncogenes (25). In the present study, we identified that IGF1R was upregulated in the NPC C666-1 cell line using a western blot assay and downregulated by miR-100, which was confirmed by luciferase assay. Knockdown of IGF1R by siRNA inhibited NPC cell proliferation. IGF1R-targeted therapy significantly suppresses pancreatic cell growth and induces apoptosis of pancreatic cancer cells (26). Consistent with our study, in ALK fusion-positive lung cancer, suppression of IGF1R expression reduced cell growth and promotes apoptosis (27). IGF1R demonstrates different functions in different cancer types. In addition, RBSP3 has been identified as a downstream target of miR-100 (11).

miR-100 may have many downstream targets and these potential targets remain to be identified. In future research, we aim to identify other targets to obtain a better understanding of NPC. Additionally, IGF1R may have other potential downstream genes and further studies should be conducted.

In summary, we identified that the overexpression of miR-100 may significantly inhibit the migration and invasion of NPC cells. IGF1R is a target of miR-100. The newly identified miR-100/IGF1R axis provides a new biomarker for developing the treatment of NPC and deepens our understanding of the mechanism of NPC tumorigenesis.

\section{Acknowledgements}

Not applicable.

\section{Funding}

No funding was received.

\section{Availability of data and materials}

The datasets used and/or analyzed during the present study are available from the corresponding author on reasonable request.

\section{Authors' contributions}

YC contributed to the conception of the study. XS contributed significantly to perform the experiment and helped to write the manuscript. XL wrote the manuscript and helped to perform the experiment. YW performed the data analyses. SY and TY helped perform the analysis with constructive discussions. All authors read and approved the final manuscript.

\section{Ethics approval and consent to participate}

The study was approved by the Ethics Committee of Weifang People's Hospital (Weifang, China). Patients provided informed consent for this study.

\section{Consent for publication}

Not applicable.

\section{Competing interests}

The authors declare that they have no competing interests.

\section{References}

1. Brennan B: Nasopharyngeal carcinoma. Orphanet J Rare Dis 1: 23-28, 2006.

2. Chang ET and Adami HO: The enigmatic epidemiology of nasopharyngeal carcinoma. Cancer Epidemiol Biomarkers Prev 15: 1765-1777, 2006

3. Bruce JP, Yip K, Bratman SV, Ito E and Liu FF: Nasopharyngeal cancer: Molecular landscape. J Clin Oncol 33: 3346-3355, 2015.

4. Torre LA, Bray F, Siegel RL, Ferlay J, Lortet-Tieulent J and Jemal A: Global cancer statistics, 2012. CA Cancer J Clin 65: 87-108, 2015

5. Thompson L: World Health Organization classification of tumours: Pathology and genetics of head and neck tumours. Ear Nose Throat J 85: 74, 2006

6. Hutvágner $\mathrm{G}$ and Zamore PD: A microRNA in a multiple-turnover RNAi enzyme complex. Science 297: 2056-2060, 2002.

7. Li Y and Kowdley KV: MicroRNAs in common human diseases. Genomics Proteomics Bioinformatics 10: 246-253, 2012.

8. O'Connell RM, Rao DS and Baltimore D: microRNA regulation of inflammatory responses. Annu Rev Immunol 30: 295-312, 2012.

9. Hayes J, Peruzzi PP and Lawler S: MicroRNAs in cancer: Biomarkers, functions and therapy. Trends Mol Med 20: 460-469, 2014.

10. Henson BJ, Bhattacharjee S, O'Dee DM, Feingold E and Gollin SM: Decreased expression of miR-125b and miR-100 in oral cancer cells contributes to malignancy. Genes Chromosomes Cancer 48: 569-582, 2009. 
11. Zheng YS, Zhang H, Zhang XJ, Feng DD, Luo XQ, Zeng CW, Lin KY, Zhou H, Qu LH, Zhang P, et al: MiR-100 regulates cell differentiation and survival by targeting RBSP3, a phosphatase-like tumor suppressor in acute myeloid leukemia. Oncogene 31: 80-92, 2012.

12. Kashuba VI, Li J, Wang F, Senchenko VN, Protopopov A, Malyukova A, Kutsenko AS, Kadyrova E, Zabarovska VI, Muravenko OV, et al: RBSP3 (HYA22) is a tumor suppressor gene implicated in major epithelial malignancies. Proc Natl Acad Sci USA 101: 4906-4911, 2004.

13. Samani AA, Yakar S, LeRoith D and Brodt P: The role of the IGF system in cancer growth and metastasis: Overview and recent insights. Endocr Rev 28: 20-47, 2007.

14. Casa AJ, Dearth RK, Litzenburger BC, Lee AV and Cui X: The type I insulin-like growth factor receptor pathway: a key player in cancer therapeutic resistance. Front Biosci 13: 3273-3287, 2008.

15. Steele-Perkins G, Turner J, Edman JC, Hari J, Pierce SB, Stover C, Rutter WJ and Roth RA: Expression and characterization of a functional human insulin-like growth factor I receptor. J Biol Chem 263: 11486-11492, 1988

16. Dziadziuszko R, Merrick DT, Witta SE, Mendoza AD, Szostakiewicz B, Szymanowska A, Rzyman W, Dziadziuszko K, Jassem J, Bunn PA Jr, et al: Insulin-like growth factor receptor 1 (IGF1R) gene copy number is associated with survival in operable non-small-cell lung cancer: A comparison between IGF1R fluorescent in situ hybridization, protein expression, and mRNA expression. J Clin Oncol 28: 2174-2180, 2010

17. Yuen JS, Cockman ME, Sullivan M, Protheroe A, Turner GD Roberts IS, Pugh CW, Werner H and Macaulay VM: The VHL tumor suppressor inhibits expression of the IGF1R and its loss induces IGF1R upregulation in human clear cell renal carcinoma. Oncogene 26: 6499-6508, 2007.

18. Katanoda $\mathrm{K}$ and Marugame T: Comparison of time trends in cancer incidence (1973-1997) in East Asia, Europe and USA, from Cancer Incidence in Five Continents Vol. IV-VIII. Jpn J Clin Oncol 37: 157-159, 2007.

19. Jia WH, Huang QH, Liao J, Ye W, Shugart YY, Liu Q, Chen LZ, Li YH, Lin X, Wen FL, et al: Trends in incidence and mortality of nasopharyngeal carcinoma over a 20-25 year period (1978/1983-2002) in Sihui and Cangwu counties in southern China. BMC Cancer 6: 178, 2006.
20. Chen R, Zhang M, Li Q, Xiong H, Liu S, Fang W, Zhang Q, Liu Z, Xu X and Jiang Q: The Epstein-Barr Virus-encoded miR-BART22 targets MAP3K5 to promote host cell proliferative and invasive abilities in nasopharyngeal carcinoma. J Cancer 8: 305-313, 2017.

21. Zhang P, Hong H, Sun X, Jiang H, Ma S, Zhao S, Zhang M, Wang Z, Jiang C and Liu H: MicroRNA-10b regulates epithelialmesenchymal transition by modulating KLF4/Notch1/E-cadherin in cisplatin-resistant nasopharyngeal carcinoma cells. Am J Cancer Res 6: 141-156, 2016.

22. Cheung CC, Lun SW, Chung GT, Chow C, Lo C, Choy KW and Lo KW: MicroRNA-183 suppresses cancer stem-like cell properties in EBV-associated nasopharyngeal carcinoma. BMC Cancer 16: 495, 2016.

23. Zhu K, He Y, Xia C, Yan J, Hou J, Kong D, Yang Y and Zheng G: MicroRNA-15a inhibits proliferation and induces apoptosis in CNE1 nasopharyngeal carcinoma cells. Oncol Res 24: 145-151, 2016.

24. Torres A, Torres K, Pesci A, Ceccaroni M, Paszkowski T, Cassandrini P, Zamboni $\mathrm{G}$ and Maciejewski R: Deregulation of miR-100, miR-99a and miR-199b in tissues and plasma coexists with increased expression of mTOR kinase in endometrioid endometrial carcinoma. BMC Cancer 12: 369, 2012.

25. Sell C, Rubini M, Rubin R, Liu JP, Efstratiadis A and Baserga R Simian virus 40 large tumor antigen is unable to transform mouse embryonic fibroblasts lacking type 1 insulin-like growth factor receptor. Proc Natl Acad Sci USA 90: 11217-11221, 1993.

26. Zhou H, Qian W, Uckun FM, Wang L, Wang YA, Chen H, Kooby D, Yu Q, Lipowska M, Staley CA, et al: IGF1 receptor targeted theranostic nanoparticles for targeted and image-guided therapy of pancreatic cancer. ACS Nano 9: 7976-7991, 2015.

27. IGF1R signaling is a therapeutic target in ALK fusion-positive lung cancer. Cancer Discov 4: 1251, 2014.

This work is licensed under a Creative Commons Attribution-NonCommercial-NoDerivatives 4.0 International (CC BY-NC-ND 4.0) License. 